\title{
Improving the Body Area Line-of-Sight Density Model: A Theoretical Study
}

\author{
Xiaodong Yang, ${ }^{1,2}$ Zhiya Zhang, ${ }^{2}$ Shuyuan Yang, \\ Qammer Hussain Abbasi, ${ }^{2,3}$ and Wei Zhao ${ }^{4}$ \\ ${ }^{1}$ School of Life Sciences and Technology, Xidian University, Xi'an 710071, China \\ ${ }^{2}$ School of Electronic Engineering, Xidian University, Xi'an 710071, China \\ ${ }^{3}$ The University of Engineering and Technology, Lahore 54890, Pakistan \\ ${ }^{4}$ School of Electro-Mechanical Engineering, Xidian University, Xian 710071, China \\ Correspondence should be addressed to Xiaodong Yang; xdyang@xidian.edu.cn
}

Received 7 February 2013; Revised 20 March 2013; Accepted 20 March 2013

Academic Editor: Duixian Liu

Copyright (c) 2013 Xiaodong Yang et al. This is an open access article distributed under the Creative Commons Attribution License, which permits unrestricted use, distribution, and reproduction in any medium, provided the original work is properly cited.

\begin{abstract}
The statistical model plays an important role in BAN radio propagation characterization. However, a traditional least-square statistical model is not necessarily the best choice when only limited samples can be collected. This paper proposes the method for improving the density model in BAN radio propagation characterization; the final PDF result validates the correctness of the method.
\end{abstract}

\section{Introduction}

In radio propagation characterization, propagation models have been developed as a suitable alterative solution to measurement campaigns [1]. Statistical models, as an essential kind of propagation model, have been broadly applied to various environments [2-8]. Recently, statistical propagation models for Body Area Networks (BANs) attract more and more attention: in [9], the authors presented a dynamic on-body channel model based on a time-variant measurement campaign at $2.45 \mathrm{GHz}$ in the $3-5 \mathrm{GHz}$ band; Fort et al. [10] measured electromagnetic waves near the torso and derived relevant statistics; in [11], Kim and Takada presented the characterization of an on-body propagation channel with a specific activity of a body on the basis of the measurement results of a male subject in a radio anechoic chamber. From all these examples, we learned that statistical models have become an indispensable part in BAN channel characterization. The statistical model, essentially, is a set of probability distributions in the sample space [12], when it is applied to channel characterization for BANs; so the specific applications are large-scale fading models and probability models of the two, the latter containing time domain characterization as well. In this paper, we mainly focus on the improvement of density models for BANs, since the results and idea are of certain reference value for the BAN channel characterization method.

\section{Density Estimation with Regression Method}

From $[13,14]$, we know that the density estimation $p(t)$ is the solution of the equation

$$
\int_{-\infty}^{\infty} \theta(x-t) p(t) d t=F(x)
$$

where $\theta(k)$ is a step function, and $F(x)$ is the probability distribution function. Meanwhile, the following conditions will be met:

$$
P(x) \geq 0, \quad \int_{-\infty}^{\infty} p(x) d x=1 .
$$




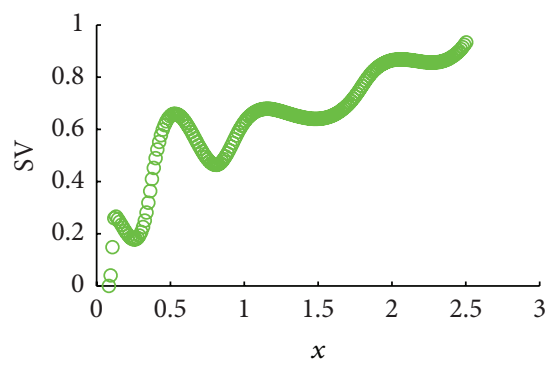

(a)

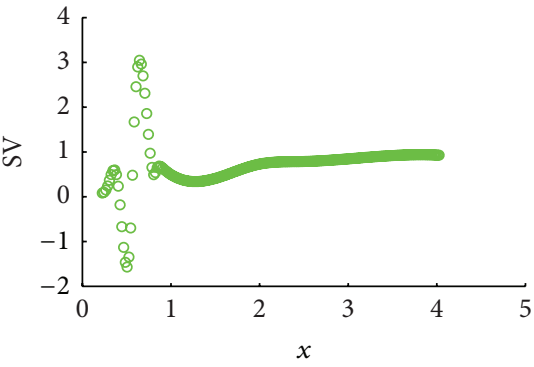

(b)

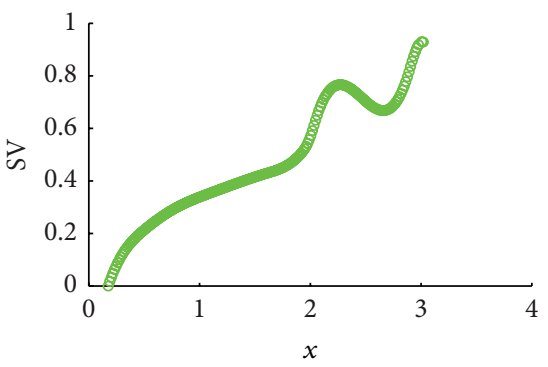

(c)

FIGURE 1: SV for RMS delay.

Problem $\int_{-\infty}^{x} p(t) d t=F(x)$
$F(x)$ is approximat

$F(x)$ is approximated by empirical distribution function $F_{l}(x)$ (Vapnik and Mukherjee, 1999)

ill - posed problem:

$A_{p}=F$,

$F$ is approximated by empirical distribution function $F_{l}(x)$

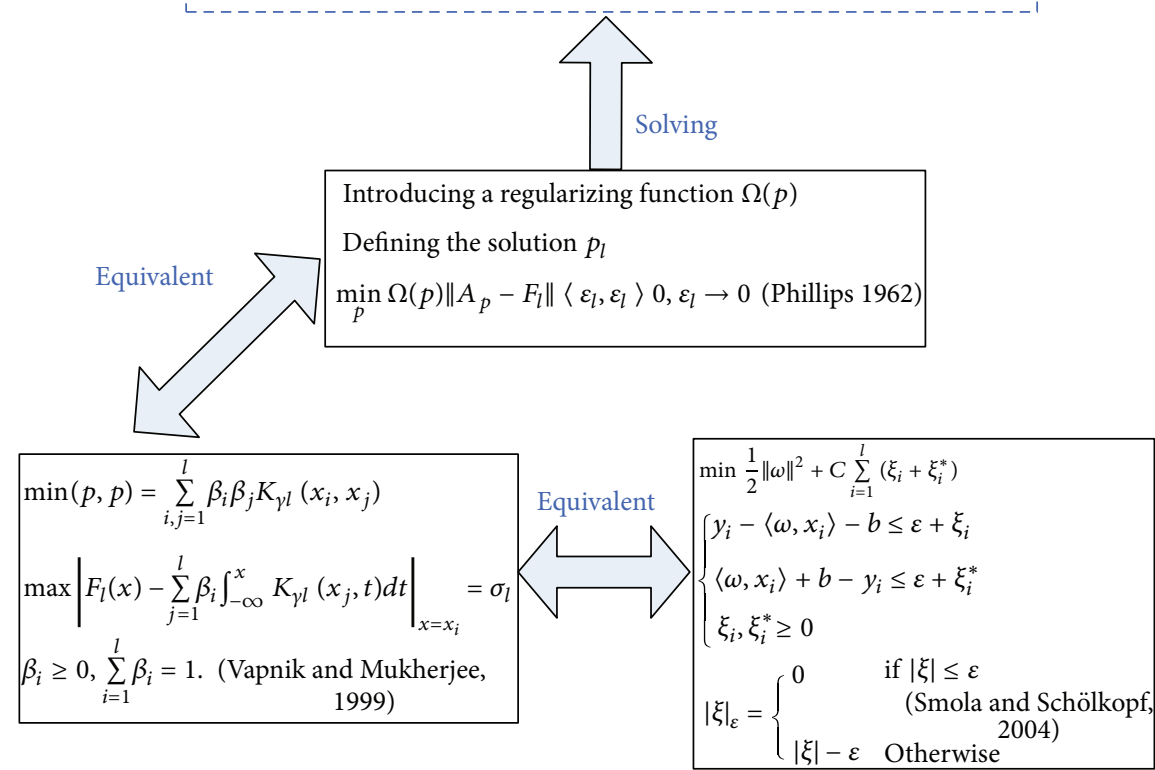

FIGURE 2: The algorithm for density estimation in BANs.

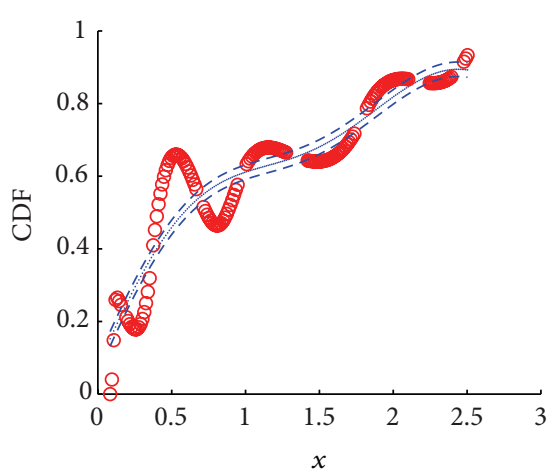

(a)

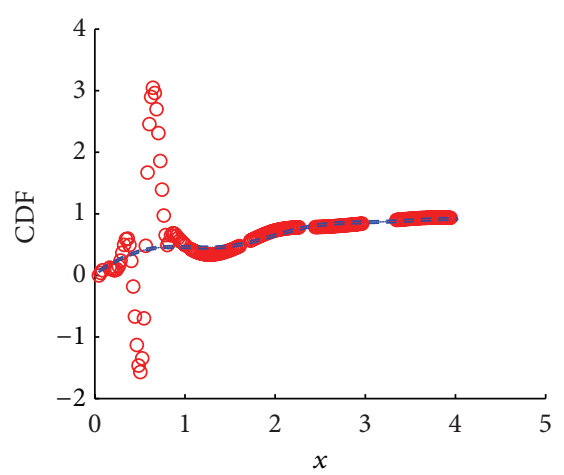

(b)

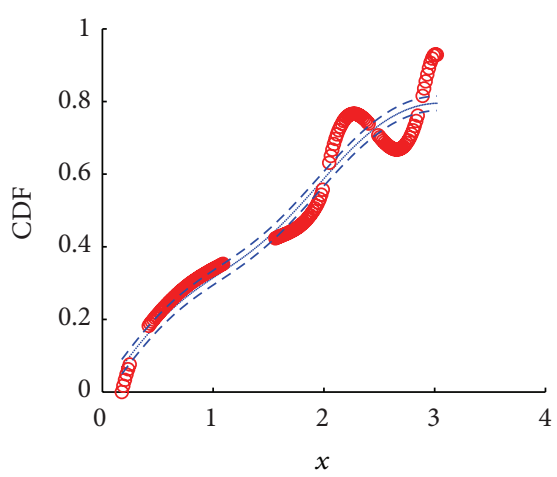

(c)

FIgURE 3: $\varepsilon$-insensitive method. 


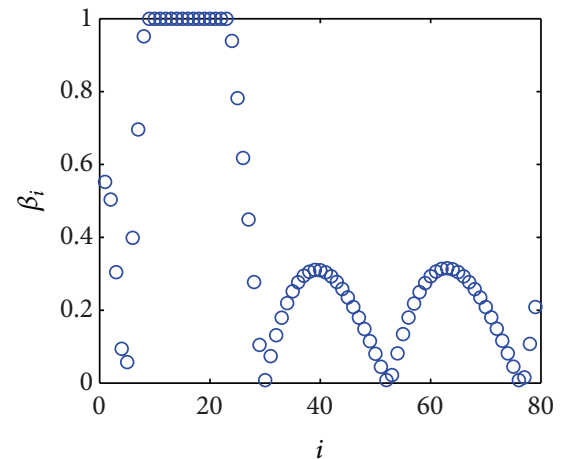

(a)

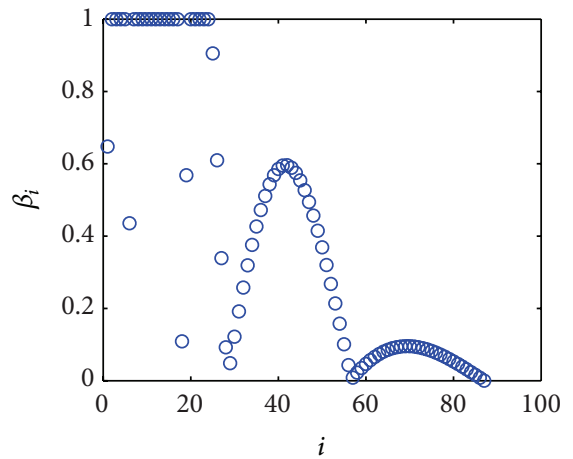

(b)

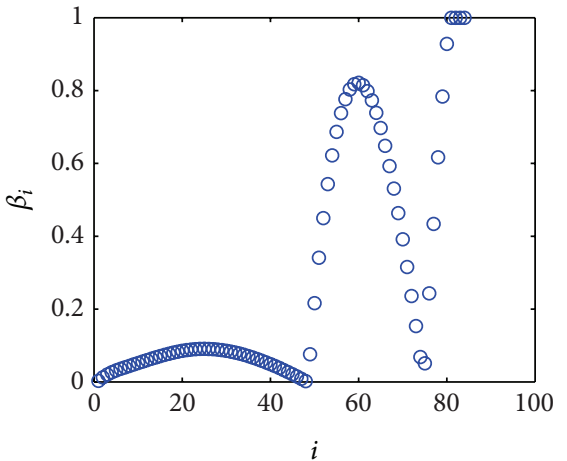

(c)

FIgURE 4: Regression coefficient.

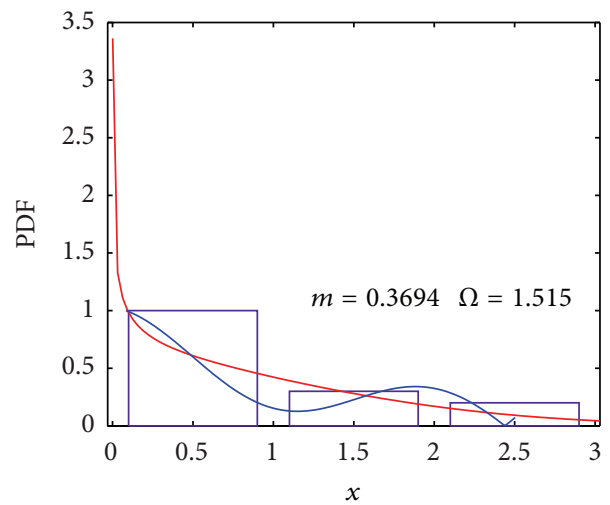

(a)

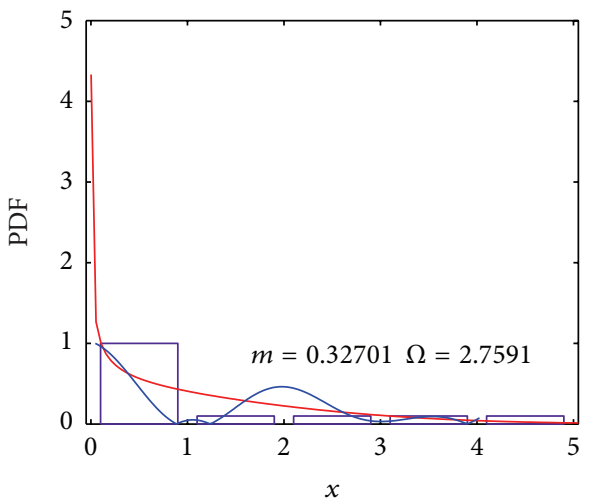

(b)

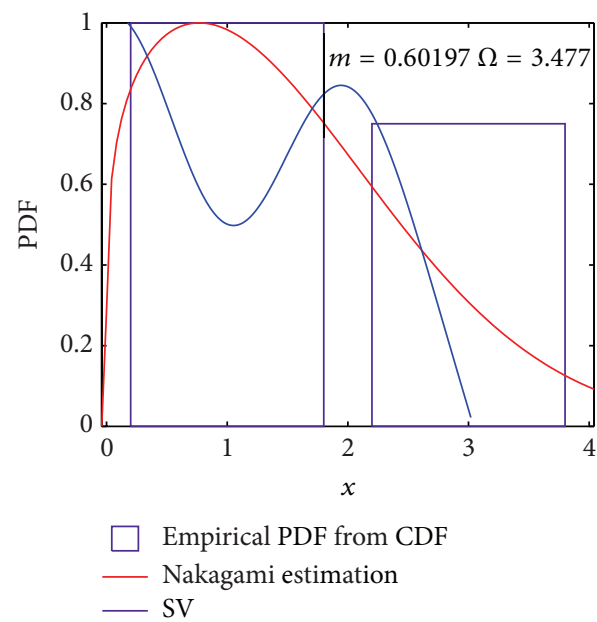

(c)

FIGURE 5: Density estimation comparisons for the RMS delay spread.

It is worth noting that, in (1), the distribution function $F(x)$ is unknown, but a random i.i.d. sample $x_{1}, \ldots, x_{l}$ is given; then the empirical distribution function is given [15]:

$$
F_{l}(x)=\frac{1}{l} \sum_{i=1}^{l} \theta\left(x-x_{i}\right),
$$

where $\theta(u)$ is a step function. We cite a common time domain parameter (root mean square delay spread) for characterizing
BAN channels as an example to build the empirical distribution function. The sampling points cover the chest, the arms, and the legs (the data were measured at the Body-Centric Lab, Queen Mary, University of London); the empirical distribution function can be seen as an approximation of actual probability density function.

The method and the train of thought for density estimation are shown in Figure 2. 
After establishing the empirical distribution function, we used the $\varepsilon$-insensitive method [16] to approximate $F_{l}(x)$. In Figure 3, the cumulative distribution function for the SV regression is shown where the $\mathrm{SV}$ is only shown in Figure 1. The $\varepsilon$-insensitive loss function $|\xi|_{\varepsilon}$ is described by [17]

$$
|\xi|_{\varepsilon}= \begin{cases}0 & \text { if }|\xi \leq \varepsilon| \\ |\xi|-\varepsilon & \text { otherwise }\end{cases}
$$

In our case, the spacing between the dotted lines is $2 \varepsilon$.

We do care about the coefficient (Figure 4) in the regression process; these coefficients $\beta_{i}$ are just the ones which would be used to do the final probability density function regression.

Figure 5 presents the density estimation results, while reasonable approximation demonstrates the feasibility and potentiality of this method. In addition, Figure 5 exhibits the traditional method as well: Nakagami distribution is very common in characterizing on-body RMS delay [18], and the results show that this density distribution is not necessarily the best choice.

\section{Conclusion}

Statistical model is an important mean of characterizing radio propagation in the BAN propagation environment and other propagation environments. In this paper, the authors try to improve the density estimation method for limited samples in an on-body line-of-sight scenario. In traditional BAN probability density models, least square technique is used to do the regression analysis; however, when the sample size is small, the precision of this method is debatable. In this paper, we proposed new mathematical mechanism to approximate probability density, the final result demonstrates the correctness of the proposed method, and this newly proposed model has extensive reference meaning for statistical radio propagation modeling.

\section{Acknowledgments}

The authors would like to thank Dr. Akram Alomainy and Professor Yang Hao, Queen Mary, University of London, UK, for comprehensive guidance; Dr Xueli Chen, Dr. Karen M. von Deneen, Professor Jimin Liang, and Professor Jie Tian, Xidian University for valuable suggestions. This work was supported by the National Natural Science Foundation of China (Grant no. 61250110542) and the Fundamental Research Funds for the Central Universities (no. K5051310004).

\section{References}

[1] A. Alomainy, Antennas and radio propagation for body-centric wireless networks [Ph.D. thesis], 2007.

[2] A. A. M. Saleh and R. A. Valenzuela, "A statistical model for indoor multipath propagation," IEEE Journal on Selected Areas in Communications, vol. 5, no. 2, pp. 128-137, 1987.
[3] J. Karedal, S. Wyne, P. Almers, F. Tufvesson, and A. F. Molisch, "A measurement-based statistical model for industrial ultrawideband channels," IEEE Transactions on Wireless Communications, vol. 6, no. 8, pp. 3028-3037, 2007.

[4] M. Hassan-Ali and K. Pahlavan, "A new statistical model for site-specific indoor radio propagation prediction based on geometric optics and geometric probability," IEEE Transactions on Wireless Communications, vol. 1, no. 1, pp. 112-123, 2002.

[5] P. Yegani and C. D. McGillem, "A statistical model for the factory radio channel," IEEE Transactions on Communications, vol. 39, no. 10, pp. 1445-1454, 1991.

[6] H. Suzuki, "A statistical model for urban radio propagation," IEEE Transactions on Communications, vol. 25, no. 7, pp. 673680, 1977.

[7] R. Kattenbach, "Statistical modeling of small-scale fading in directional radio channels," IEEE Journal on Selected Areas in Communications, vol. 20, no. 3, pp. 584-592, 2002.

[8] D. Cassioli, M. Z. Win, and A. F. Molisch, "The ultra-wide bandwidth indoor channel: from statistical model to simulations," IEEE Journal on Selected Areas in Communications, vol. 20, no. 6, pp. 1247-1257, 2002.

[9] R. D'Errico and L. Ouvry, "A statistical model for on-body dynamic channels," International Journal of Wireless Information Networks, vol. 17, no. 3-4, pp. 92-104, 2010.

[10] A. Fort, C. Desset, P. de Doncker, P. Wambacq, and L. van Biesen, "An ultra-wideband body area propagation channel model-from statistics to implementation," IEEE Transactions on Microwave Theory and Techniques, vol. 54, no. 4, pp. 18201826, 2006.

[11] M. Kim and J. I. Takada, "Statistical model for 4.5-GHz narrowband on-body propagation channel with specific actions," IEEE Antennas and Wireless Propagation Letters, vol. 8, pp. 1250-1254, 2009.

[12] P. McCullagh, "What is a statistical model?" The Annals of Statistics, vol. 30, no. 5, pp. 1225-1267, 2002.

[13] E. Parzen, "On estimation of a probability density function and mode," Annals of Mathematical Statistics, vol. 33, no. 3, pp. 10651076, 1962.

[14] B. W. Silverman, "Density Estimation for Statistical and Data Analysis," http://ned.ipac.caltech.edu/level5/March02/Silverman/paper.pdf.

[15] V. Vapnik and S. Mukherjee, "Support vector method for multivariate density estimation," Neural Information Processing Systems, pp. 659-665, 1999.

[16] A. J. Smola and B. Schölkopf, "A tutorial on support vector regression," Statistics and Computing, vol. 14, no. 3, pp. 199-222, 2004.

[17] D. L. Phillips, "A technique for the numerical solution of certain integral equations of the first kind," Journal of the Association for Computing Machinery, vol. 9, pp. 84-97, 1962.

[18] A. Sani, A. Alomainy, G. Palikaras et al., "Experimental characterization of UWB on-body radio channel in indoor environment considering different antennas," IEEE Transactions on Antennas and Propagation, vol. 58, no. 1, pp. 238-241, 2010. 

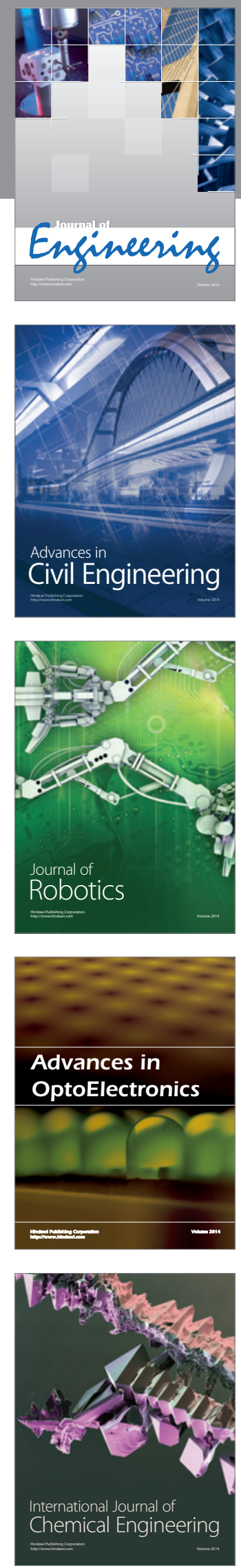

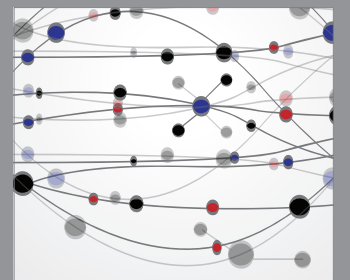

The Scientific World Journal
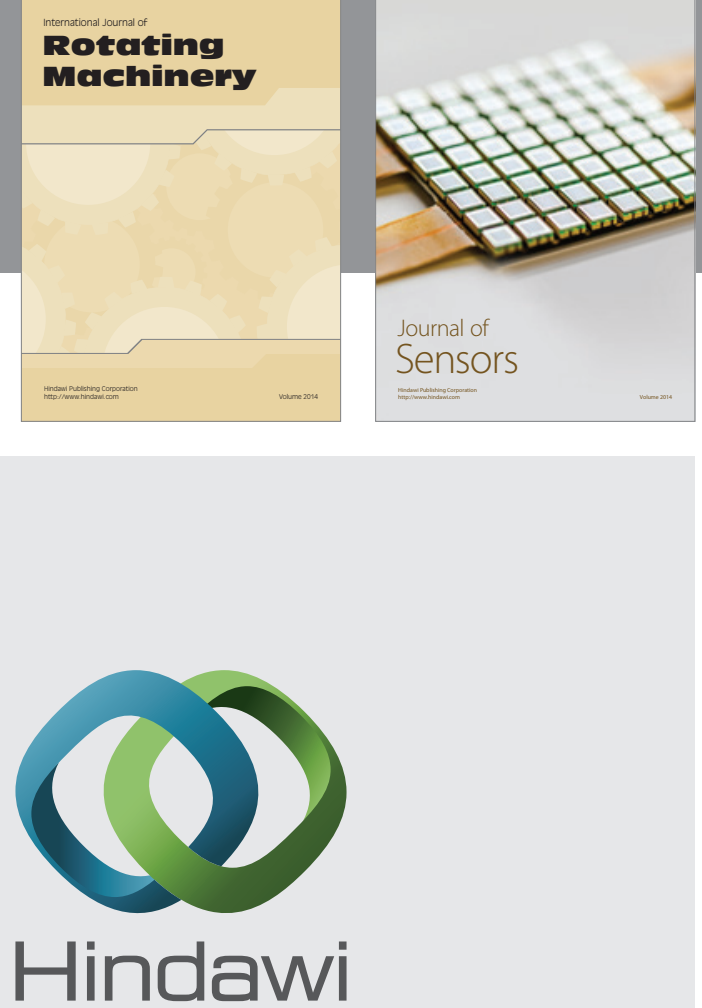

Submit your manuscripts at http://www.hindawi.com
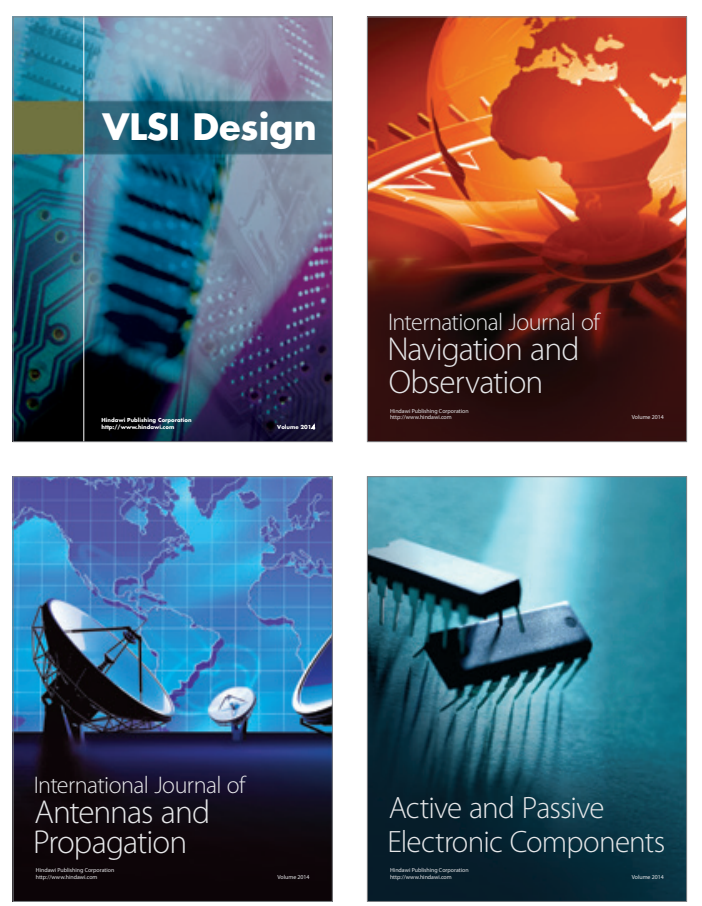
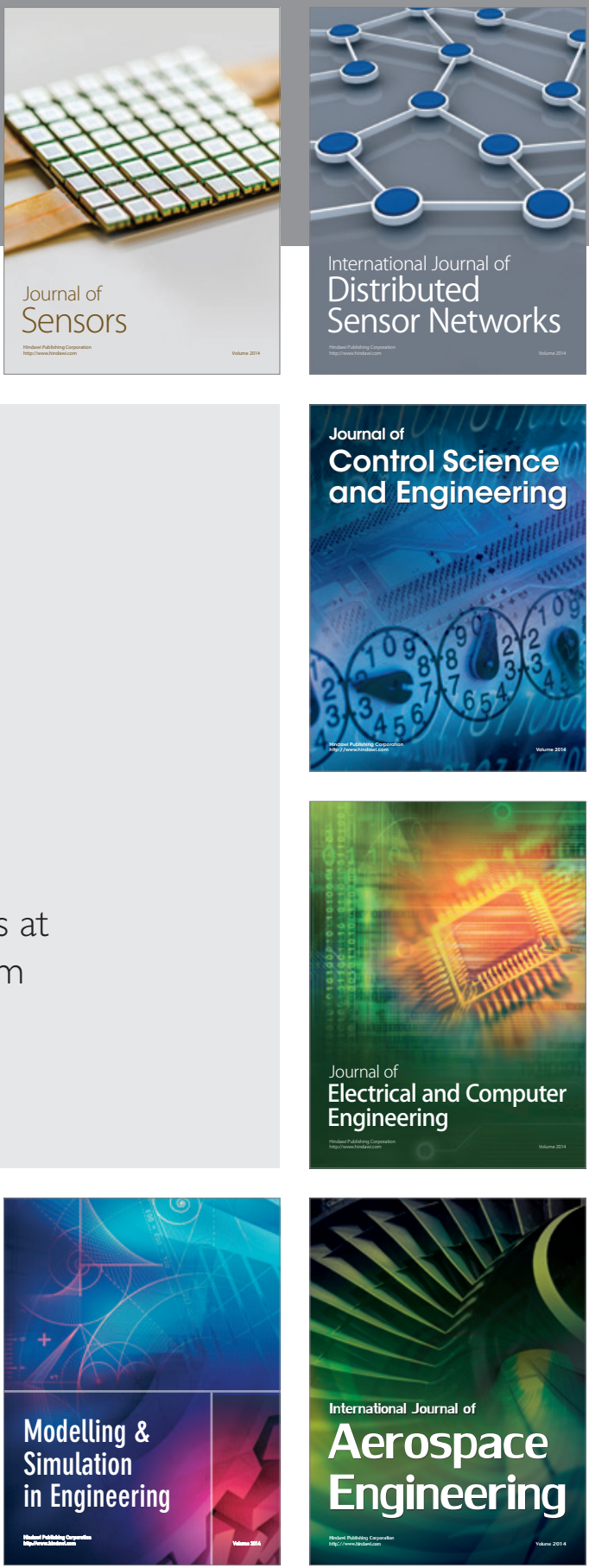

Journal of

Control Science

and Engineering
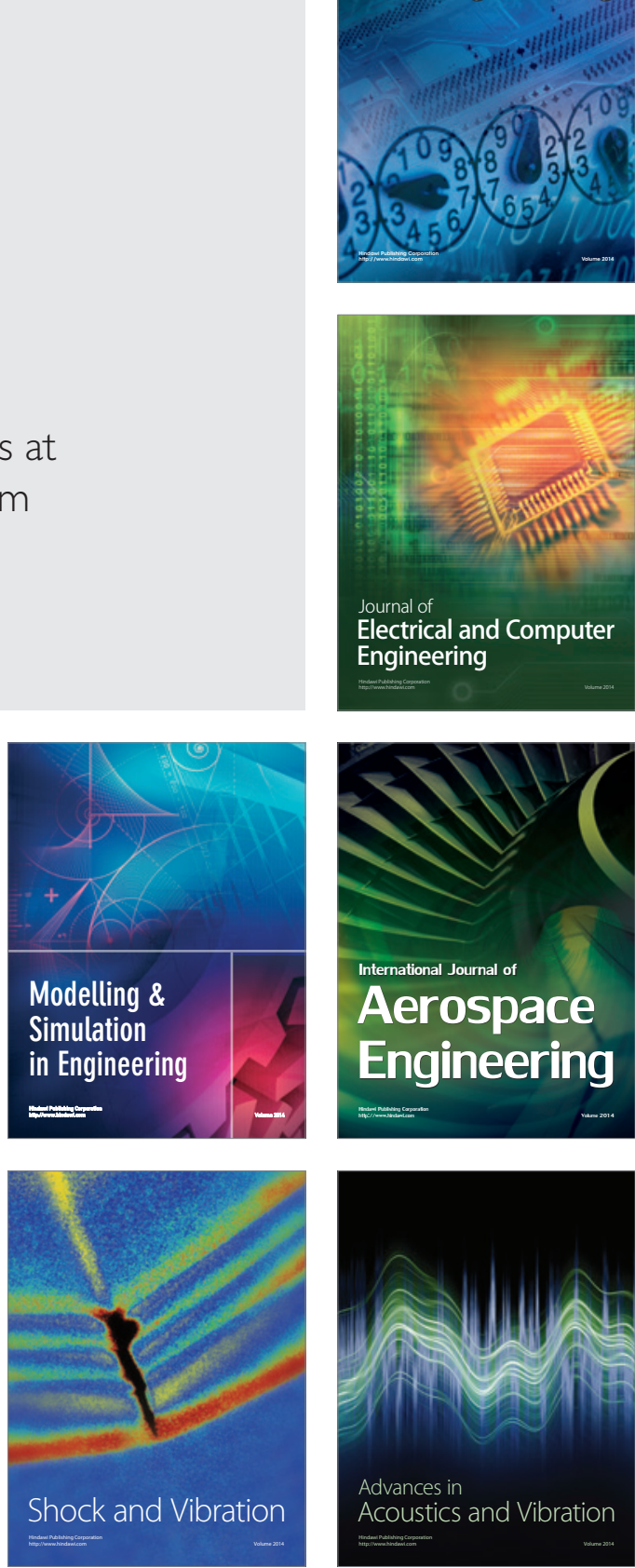